\title{
CHARACTERIZATIONS OF COMPACTNESS OF THE INTERVAL TOPOLOGY IN SEMILATTICES
}

\author{
T. B. MUENZENBERGER AND R. E. SMITHSON
}

ABSTRACT: In a semilattice two necessary and sufficient conditions for the interval topology to be compact are established. One is in terms of the fixed point property for increasing functions on the semilattice, and the other is in terms of completeness of the semilattice.

Several years ago L. E. Ward, Jr. stated a characterization of the fixed point property for increasing functions on semilattices [5]. Subsequently the authors of the present paper detected a flaw in the argument. Since the theorem is of some importance [2] and has not been independently subsumed in the literature, it seems desirable to publish a corrected and improved version of the theorem.

Let $(X, \leq)$ be a partially ordered set. We write

$$
L(x)=\{y \in X \mid y \leq x\} \text { and } M(x)=\{y \in X \mid x \leq y\}
$$

for each $x \in X$. The sets $L(x)$ for $x \in X$ are called the lower sets. The pair $(X, \leq)$ is a semilattice if and only if the infimum of $\{x, y\}$ exists for each $x, y \in X$; the infimum will be denoted by $x \wedge y$. If $(X, \leq)$ and $(X, \geq)$ are semilattices, then $(X, \leq)$ is a lattice. A subset $C$ of $X$ is a chain if and only if any two elements of $C$ are comparable under $\leq$. A semilattice $(X, \leq)$ is complete if and only if the infimum of each nonempty subset $A$ of $\bar{X}$ exists; the infimum will be denoted by $\wedge A$. The supremum of $A$, when it exists, will be denoted by $\bigvee A$.

We say that $(X, \leq)$ is closed above if and only if every nonempty chain in $X$ has a supremum. The interval topology for $(X, \leq)$ is that topology generated by taking all of the sets $L(x)$ and $M(x)$ for $x \in X$ as a subbasis for the closed sets. A function $f: X \rightarrow X$ is increasing if and only if $x \leq y$

Received by the editors August 15, 1973.

AMS (MOS) subject classifications (1970). Primary 54F05.

Key words and phrases. Fixed point property, increasing functions, lattices, semilattices. 
implies $f(x) \leq f(y)$ for each $x, y \in X$. A fixed point of $f: X \rightarrow X$ is a point $x \in X$ such that $x=f(x)$. Finally, $X$ has the fixed point property (f.p.p.) for increasing functions if and only if every increasing function $f: X \rightarrow X$ has a fixed point.

The first lemma is a corollary of a fixed point theorem of Abian, Brown, and Smithson [3], but the proof is simple and is therefore included.

Lemma 1. If $(X, \leq)$ is a partially ordered set with least element $e$ and if $X$ is closed above, then $X$ has the f.p.p. for increasing functions.

Proof. Consider $A=\{x \in X \mid x \leq f(x)\}$ where $f: X \rightarrow X$ is increasing. Then $e \in A$. Let $C$ be a maximal chain in $A$ and let $s=\bigvee C$. For each $x \in C$, $x \leq f(x) \leq f(s)$, and so $s \leq f(s)$. Applying $f$ to the last inequality, $f(s) \leq$ $f(f(s))$. Thus $f(s) \in A$, and therefore $f(s) \in C$ by the maximality of $C$. Hence $s=f(s)$ is a fixed point of $f$.

The examples we have are suprisingly simple. Let $X=[0,1] \cup\{a, b\}$ where we take the usual order $\leq$ on the unit interval $[0,1]$ and define $0<$ $a, b<x$ for all $x \in(0,1]$. Then $(X, \leq)$ satisfies the hypotheses and therefore the conclusion of Lemma 1 . Also $(X, \leq)$ is a semilattice, but $X$ is neither complete, nor a lattice, nor compact in the interval topology. Thus Theorem 3 and Lemma 2 of [5] are incorrect. Moreover, this example shows that it is necessary to assume that the lower sets are lattices in Lemma 4 and Theorem 5 following. Now every nonempty chain in the partially ordered set $(X, \preceq)$, where $\leq$ is the dual order, has an infimum, and $(X, \preceq)$ has a greatest element. Therefore $(X, \preceq)$ has the f.p.p. for increasing functions by the dual of Lemma 1 . More simply, $f$ is increasing with respect to $\preceq$ if and only if $f$ is increasing with respect to $\leq$.

Thus in the next lemma it is necessary to have a semilattice structure or at least to assume the condition used in [4].

For the remainder of the paper, let $(X, \leq)$ be a semilattice, and give $X$ the interval topology. The following lemma was proved in [4].

Lemma 2. If $X$ has the f.p.p. for increasing functions, then $X$ is closed above.

The next lemma was proved in [5].

Lemma 3. The lower sets are compact if and only if $X$ is complete.

Lemma 4. If $X$ has the f.p.p. for increasing functions and if the lower sets are lattices, then $X$ is complete. 
Proof. First, let $x_{0} \in X$. Then $L\left(x_{0}\right)$ is a complete lattice. For if $f: L\left(x_{0}\right) \rightarrow L\left(x_{0}\right)$ is increasing, then the function $\hat{f}: X \rightarrow X$, defined by $\hat{f}(x)=$ $f\left(x \wedge x_{0}\right)$ for each $x \in X$, is increasing. Hence $\hat{f}$ has a fixed point. But any fixed point of $\hat{f}$ is in $L\left(x_{0}\right)$, and $\hat{f}=f$ on $L\left(x_{0}\right)$. Thus $f$ has a fixed point. Finally, $L\left(x_{0}\right)$ is a lattice, and hence, $L\left(x_{0}\right)$ is complete by a theorem of Davis [1].

Now let $\varnothing \neq A \subset X$ and let $x_{0} \in A$. Define $A^{\prime}=\left\{a \wedge x_{0} \mid a \in A\right\}$. If $x$ is a lower bound of $A^{\prime}$, then $x$ is a lower bound of $A$. On the other hand, if $x$ is a lower bound of $A$, then $x \leq a$ for all $a \in A$. In particular, $x \leq x_{0}$, and so $x \leq a \wedge x_{0}$ for all $a \in A$. So $x$ is a lower bound of $A^{\prime}$. Finally, $A^{\prime} \subset$ $L\left(x_{0}\right)$, and so $A^{\prime}$ has an infimum in $L\left(x_{0}\right)$ which is an infimum of $A$ in $X$ by the above.

Theorem 5. If $X$ has the f.p.p. for increasing functions and if the lower sets are lattices, then $X$ is compact.

Proof. By Alexander's lemma and Lemmas 3 and 4, it suffices to show that any collection $\mathcal{F} \subset\{M(x) \mid x \in X\}$ with f.i.p. has nonempty intersection. We proceed by induction. Assume that $m$ is a cardinal number such that if $\mathcal{F} \subset\{M(x) \mid x \in X\}$ has f.i.p. and if $|\mathcal{F}|=\operatorname{Card}(\mathcal{F})<m$, then $\bigcap^{\mathcal{F}} \neq \varnothing$. Next let $\Gamma$ be the smallest ordinal such that $|\Gamma|=m$. Let $\mathcal{F} \subset\{M(x) \mid x \in X\}$ have f.i.p. and satisfy $|\mathcal{F}|=m$. Note that each subcollection of $\mathcal{F}$ also has f.i.p. Since $|\mathcal{F}|=m=|\Gamma|$, there is a one to one function $i$ from $\mathcal{F}$ onto $\Gamma$. For each $\alpha \in \Gamma$, write $F_{a}=i^{-1}(\alpha)$. Now if $\alpha \in \Gamma$, then $|a|<m$, and so $\bigcap\left\{F_{\gamma} \mid \gamma \leq \alpha\right\} \neq \varnothing$. For each $\alpha \in \Gamma$, we may set $z_{\alpha}=\bigwedge \bigcap\left\{F_{\gamma} \mid \gamma \leq \alpha\right\}$ since $X$ is complete by Lemma 4. The set $C=\left\{z_{\alpha} \mid a \in \Gamma\right\}$ is a chain in $X$. So we may let $z_{0}=\bigvee C$ by Lemma 2. For each $a \in \Gamma$, there is $x_{a} \in X$ such that $F_{a}=M\left(x_{a}\right)$. Then $x_{\alpha} \leq z_{\alpha} \leq z_{0}$ for each $\alpha \in \Gamma$. Hence, $z_{0} \in \bigcap\left\{F_{\alpha} \mid a \in \Gamma\right\}=$ $\bigcap^{\mathcal{F}}$. Consequently, any collection $\mathcal{F} \subset\{M(x) \mid x \in X\}$ with f.i.p. and satisfying $|\mathcal{F}|=m$ has nonempty intersection. This completes the induction. Thus $X$ is compact in the interval topology.

The proof of the next lemma is not difficult.

Lemma 6. If $X$ is complete, then every nonempty subset of $X$ which is bounded above has a supremum, and the lower sets are lattices.

Theorem 7. The following are equivalent:

(i) $X$ is compact;

(ii) $X$ is closed above and complete; 
(iii) $X$ has the f.p.p. for increasing functions and the lower sets are lattices.

Proof. For (i) implies (ii), assume that $X$ is compact. Then $X$ is complete by Lemma 3. Let $C$ be a nonempty chain in $X$. Then $\{M(x) \mid x \in C\}$ has f.i.p. since $C$ is a chain. So $\bigcap\{M(x) \mid x \in C\} \neq \varnothing$ since $X$ is compact. Thus $C$ is bounded above. Apply Lemma 6. For (ii) implies (iii), assume that $X$ is closed above and complete. Then $X$ has a least element; namely, $e=\bigwedge X$. Then Lemma 1 implies that $X$ has the f.p.p. for increasing functions. Lemma 6 implies that the lower sets are lattices. Finally, (iii) implies (i) is exactly Theorem 5.

\section{REFERENCES}

1. A. C. Davis (A. C. Morel), A characterization of complete lattices, Pacific J. Math. 5 (1955), 311-319. MR 17, 574.

2. T. B. Muenzenberger and R. E. Smithson, Fixed point structures, Trans. Amer. Math. Soc. 184 (1973), 153-173.

3. R. E. Smith son, Fixed points of order preserving multifunctions, Proc. Amer. Math. Soc. 28 (1971), 304-310. MR 43 \# 114.

4. - Fixed points in partially ordered sets, Pacific J. Math. 45 (1973), 363-367.

5. L. E. Ward, Jr., Completeness in semi-lattices, Canad. J. Math. 9 (1957), 578-582. MR 19, 938.

DEPARTMENT OF MATHEMATICS, UNIVERSITY OF WYOMING, LARAMIE, WYOMING 82071

Current address (T. B. Muenzenberger): Department of Mathematics, Kansas State University, Manhattan, Kansas 66506

Current address (R. E. Smithson): Department of Mathematics, University of Houston, Houston, Texas 77004 